most comfortable. METHODS/STUDY POPULATION: We have used several strategies to enable investigators to gain input from stakeholders in rural and frontier areas. If the research focuses on rural populations, we hold the Studio at a central location, usually at a restaurant in a private room, if this is available. If the investigator wants to hear from both rural and urban residents, we use videoconferencing via Skype or FaceTime when individuals have enough bandwidth to support it and/or feel comfortable using this technology. For those who have dial-up or no internet access, we provide a conference call line Trusting relationships are essential to creating a safe space in which stakeholders from communities facing health disparities can provide consultations to researchers. When an investigator wishes to consult with stakeholders from one racial/ethnic community, we contract with a leader or trusted member of that community to recruit appropriate stakeholders. The Studio is co-facilitated by a CCET staff member and a community leader in the community's preferred language, with the leader translating for the CCET staff member. For Studios that involve stakeholders from multiple communities and that are conducted in English, we provide translators, if appropriate. Stakeholders using translation may be present in the room with other Studio participants or may be on the phone. RESULTS/ANTICIPATED RESULTS: Of the 35 Studios we have held, five have been held in rural locations and another five have included one or more rural/frontier stakeholders participating via phone or videoconferencing. Six Studios have been co-facilitated with community leaders and four others have included translators. Almost all Studios we have held in English have included individuals representing diverse communities. Anonymous surveys completed at the end of Studios show that participants report the following on 5-point Likert scales:. The facilitator managed the allotted time so that my voice was heard (67\% strongly agree; $33 \%$ agree). The relevant experts were present at the Studio (78\% strongly agree; $22 \%$ agree). I was satisfied with the Studio session (78\% strongly agree; $22 \%$ agree). The Studio process was worth my time (89\% strongly agree; $11 \%$ agree). The feedback provided by the community experts will improve the research project (68\% strongly agree; $44 \%$ agree). Participants were also asked what they felt was their contribution to the research project. Among the most common themes were: increased researcher's understanding of the community, increased researcher's sensitivity to the community, provided feedback on the feasibility of the project, provided ideas on recruiting research participants, provided ideas for how to use the project results to benefit the community, and provided ideas on how to inform the community about the project. All participants said that they would participate in a Studio again. DISCUSSION/SIGNIFICANCE OF IMPACT: Studies at all stages in the research life cycle can be strengthened through consultations with community experts. These stakeholders can inform needs assessments, provide input on study design, supply critical information on supports and barriers to research participation, review study instruments for readability and cultural appropriateness, provide feedback on recruitment and educational materials, and inform dissemination of research results, among others. These consultations provide the most benefit to researchers when they include the voices of as broad a range of stakeholders as possible. We have shown that it is feasible to include stakeholders who live in rural and frontier areas in Studio consultations. We also have developed successful methods for holding Studio consultations with stakeholders who are members of communities facing health disparities and who speak multiple languages. This expanded representation in Community Engagement Studios strengthens research studies.

\section{Addressing Community Health Needs through Community Engagement Research Advisory Boards} Christi Patten, $\mathrm{PhD}^{1}$, Monica L. Albertie, $\mathrm{MHA}^{1}$, Chara A. Chamie, $\mathrm{MPH}^{1}$, Tabetha A. Brockman, $\mathrm{MA}^{1}$, Mary Gorfine ${ }^{1}$, Rosa Nicholas ${ }^{1}$, Martha J. Bock, BA ${ }^{1}$, Janet M. Okamoto, $\mathrm{PhD}^{1}$, Sumedha G. Penheiter, PhD $^{1}$ and Joyce E. Balls-Berry, PhD $^{1}$ ${ }^{1}$ Mayo Clinic

OBJECTIVES/SPECIFIC AIMS: Over $80 \%$ of CTSA programs have a community advisory board (CAB), an effective strategy to increase community engagement (CE) in research. Little is known about how the research discussed with CABs aligns with community priorities (i.e., bi-directionality). This program evaluation assessed the health topics presented by researchers to the CABs linked to our CE Program at all three Mayo Clinic sites (MN, AZ, and FL) for relevance to local community needs. METHODS/STUDY POPULATION: Two coders classified Mayo researcher presentations to our CABs from 2014-2018 for relevance to needs identified in the local 2013 and/or 2016 County Health Needs Assessments and specific topic(s); with high levels of agreement (Kappa=0.90). RESULTS/ANTICIPATED RESULTS: Overall, of the 65 presentations $41(63 \%)$ addressed one or more local health needs $(47 \% \mathrm{MN}$, $60 \% \mathrm{FL}, 80 \% \mathrm{AZ}$ ). Cross-cutting health topics addressed at 2 sites were physical activity/obesity/nutrition and mental health. DISCUSSION/SIGNIFICANCE OF IMPACT: Findings were shared with our CABs to obtain input on future directions. The FL and AZ $\mathrm{CABs}$ are systematic in seeking out or initiating research projects that address local health needs, an approach the MN site is interested in adopting. Ultimately, it is important to demonstrate improved health outcomes with CTSA-based CE research strategies. Understanding community health needs and depth of researchers in those areas may help to focus priorities for demonstrating such outcomes.

3191

\section{Adolescent Substance Use: School and Community Perspectives on School-Based Interventions}

Sycarah Fisher

University of Kentucky Center for Clinical and Translational Science

OBJECTIVES/SPECIFIC AIMS: Fifty percent of adolescents have tried an illicit drug and $70 \%$ have tried alcohol by the end of high school. Further, despite 7-9\% of youth 12-17 meeting criteria for a substance use disorder only 1 in 10 actually receive it. Screening, Brief Intervention, and Referral to Treatment (SBIRT) is an evidence based process that facilitates early identification and treatment for adults and adolescents in community (primary care) facilities. Despite the documented effectiveness of SBIRT, no research has examined the implementation of SBIRT in school settings by schoolbased mental health personnel. The purpose of the present study was to identify facilitators and barriers to SBIRT implementation by school-based personnel in secondary schools. METHODS/STUDY POPULATION: Participants included 30 school and community service providers including: teachers, school counselors, school psychologists, school administrators (principals and central office staff), city council members, school board members, community mental health services providers as well as state level individuals from the department of Adolescent Substance Use and the Office of Drug Control Policy. Interview guides were developed using the 
Consolidated Framework for Implementation Research (CFIR) to identify facilitators and barriers regarding the following: inner setting, outer setting, individuals involved, and intervention (SBIRT). The six-phase framework of Thematic Analysis (TA) was employed to analyze the data. We specifically used the deductive method to analyze the data with a pre-determined theory in mind (CFIR) to move to hypothesis building, and coding the data. RESULTS/ ANTICIPATED RESULTS: Contrary to research conducted outside of the schools under the auspices that schools do not have the time or interest in providing school-based substance use interventions, several themes emerged identifying a receptivity, willingness, and eagerness to provide these services. Specifically, school-based mental health professionals (i.e., school counselors, school psychologists) being aware of adolescent substance use in their schools, but not knowing how to appropriately handle such disclosures. Further, school-based mental health personnel indicated that they would want additional training on how to identify and provide services to adolescents with substance use needs. School-based administrators also indicated a receptivity to addressing substance use with an acknowledgement that schools would need to move from a punitive model for substance use infractions to a treatment model. Some identified barriers to implementation included lack of awareness of community treatment settings for referrals and anonymity or lack thereof of substance use screening. DISCUSSION/ SIGNIFICANCE OF IMPACT: While the data analyzed come from a limited sample in one school district, the present study found that schools could be potential settings for the early identification and intervention of adolescent substance use. Findings from this study contribute to our understanding of school and community receptivity to school-based interventions. Future research should identify training needs of school-based mental health personnel to assist in the early identification and prevention of substance use disorders.

3567

An Analysis of Current Trends in Inclusion of Historically Underrepresented Populations in Clinical Trials: Women and Geriatrics

Jacqueline Chen ${ }^{1}$, Kaitlyn Park ${ }^{1}$, Sun Young Uhm ${ }^{1}$, Amelia Spinrad ${ }^{1}$, Apurva Uniya ${ }^{1}$, Nancy Pire-Smerkanich ${ }^{1}$ and Eunjoo Pacifici ${ }^{1}$

${ }^{1}$ University of Southern California

OBJECTIVES/SPECIFIC AIMS: Clinical trials (CTs) play an important role in developing new treatments, expanding or refining treatments that are already available, and/or identifying behavioral changes that can prolong or improve the lives of subjects. CTs are also conducted to understand normal human physiology, pathophysiology, and factors associated with health outcomes. Results from CTs are then used to determine the safety and efficacy of medications or treatment. CT participants should reflect the diversity of those receiving the treatments because, exclusion of specific populations in CTs may potentially result in knowledge gaps for clinicians and regulators. Historically, women and geriatrics have been underrepresented as CT participants. For women, this is the result of Food and Drug Administration (FDA) action in 1977 which restricted women with childbearing potential from participating in phase I and early phase II CTs after thousands of birth defects resulted from thalidomide usage during pregnancy. While the U.S. Government Accountability Office's 1992 and 2001 reports documented an increased female inclusion in later stages of CTs, earlier phases of CTs were still lacking. Likewise, older adults and geriatrics have been excluded in CTs arbitrarily or to avoid adverse events associated with drug-drug interactions and comorbidities. Over the past few decades, the FDA has worked to address this issue and increase diversity and transparency in CTs. In 2015, the FDA's Action Plan for Food and Drug Administration Safety and Innovation Act (FDASIA) Section 907 called for improved CT inclusion and reporting of demographic subgroups (sex, age, race, and ethnicity), highlighting three priority areas: quality, participation, and transparency. This research examines the current state of female inclusion in phase I and II CTs (2016 to 2017) and geriatric inclusion in phase III CTs (2010 to 2017). METHODS/STUDY POPULATION: To assess female representation in phase I and II CTs, data from 2016 CTs was extracted from clinicaltrials.gov. The average percentage of male and female participation in trials recruiting for males and females was determined; CTs conducted in only males or females (due to sex specific disease states) were excluded. The data was further differentiated into investigatorinitiated and industry-sponsored trials to determine any differences in sex representation. Data from 2017 CTs on clinicaltrials.gov will be extracted and analyzed as well as 2016 to 2017 data from FDA novel drug approvals. To assess geriatric representation in phase III CTs, geriatric subsections of drug labels from novel drug applications approved between 2010 to 2017 were assessed for geriatricspecific information based on four areas: 1) reporting of CT including geriatrics, 2) reporting of percentage of CT participants ages $75+$, 3) providing geriatric dosage recommendations, 4) determining product safety and efficacy for geriatrics. RESULTS/ANTICIPATED RESULTS: It is mandatory that all US CTs are registered on clinicaltrials.gov with the exception of Phase I studies, and results posted within 1 year of CT completion. In 2016, 916 phase I and 713 phase II CTs were registered on clinicaltrials.gov. Of these registered CTs, $4 \%$ of phase I and $9 \%$ of phase II CTs posted results. Of these, phase I studies included more males than females. Of these, phase I studies showed higher percentage of males (58\%) than females (42\%). In phase I/II, phase II, and phase II/III CTs, females were represented at a higher levels than males by $8-20 \%$ (Table 1 ). Phase I industry-sponsored and investigator-initiated trials and phase II/III investigator-initiated trials included less females than males (Table 2); all other types of CTs had more female than male subjects (Table 2). Preliminary findings will be expanded to include 2017 CTs and a wider pool of clinical trials will include all those associated with FDA novel drugs approved in 2016 and 2017. Of the 250 labels of novel drugs approved from 2010 to 2017 assessed for geriatric inclusion, $74 \%$ reported a CT including geriatrics, and $55 \%$ reported including CT participants ages $75+$. Further, $31 \%$ provided geriatric dosage recommendations and $62 \%$ indicated insufficient evidence to determine product safety/efficacy for geriatrics (Figure 1). There was no consistent increase following the 2015 implementation of FDASIA section 907 in any of the four areas examined (Figure 2). Labels providing geriatric dosage recommendations were consistently the least fulfilled area across all years analyzed (Figure 3). DISCUSSION/SIGNIFICANCE OF IMPACT: A lack of inclusion of specific populations in CTs can lead to serious complications. For example, in 2013, the FDA required a lower recommended dose for women for drugs containing the sedativehypnotic zolpidem (i.e. Ambien) due to persisting next morning drowsiness; the FDA arbitrarily recommended the dosage be halved from $10 \mathrm{mg}$ to $5 \mathrm{mg}$ as it found that women appeared to eliminate zolpidem from their bodies more slowly than men. Additionally, $\$ 35.7$ million is spent annually on hospitalization from adverse drug reactions in the elderly. And, although government acts and initiatives have called for greater inclusion of certain populations like females and geriatrics in CTs, there is no penalty for exclusion. Problems like these may be avoided if these specific populations 